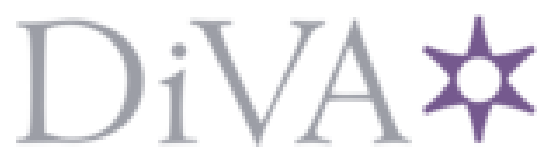

http://www.diva-portal.org

This is the published version of a paper published in Aerospace Medicine and Human Performance.

Citation for the original published paper (version of record):

Rosa, E., Grönkvist, M., Kölegård, R., Dahlström, N., Knez, I. et al. (2021)

Fatigue, emotion and cognitive performance in simulated long-duration single-piloted flight missions

Aerospace Medicine and Human Performance, 92(9): 710-719

https://doi.org/10.3357/AMHP.5798.2021

Access to the published version may require subscription.

N.B. When citing this work, cite the original published paper.

Permanent link to this version:

http://urn.kb.se/resolve?urn=urn:nbn:se:hig:diva-35491 


\section{Fatigue, Emotion, and Cognitive Performance in Simulated Long-Duration, Single-Piloted Flight Missions}

Eduardo Rosa; Mikael Gronkvist; Roger Kolegard; Nicklas Dahlstrom; Igor Knez; Robert Ljung; Johan Willander

BACKGROUND: Fatigue of air force pilots has become an increasing concern due to changes in mission characteristics. In the current study we investigated fatigue, emotions, and cognitive performance in a simulated 11-h mission in the 39 Gripen fighter aircraft.

MEthods: A total of 12 subjects were evaluated in a high-fidelity dynamic flight simulator for 12 consecutive hours. Perceived fatigue was measured by the Samn-Perelli Fatigue Index (SPFI). Emotions were assessed with the Circumplex Affect Space. Cognitive performance was assessed by five cognitive tasks.

RESULTS: Significant increase in self-reported fatigue, general decrease in two positive emotional states, as well increase of one negative emotional state occurred after approximately $7 \mathrm{~h}$ into the mission. Self-reported fatigue negatively correlated with enthusiasm and cheerfulness $\left(r^{\prime}=-0.75 ;-0.49\right.$, respectively $)$ and positively correlated with boredom and gloominess $\left(r^{\prime}=-0.61 ; r^{\prime}=-0.30\right.$, respectively). Response time in the low-order task negatively correlated with enthusiasm, cheerfulness and calmness $\left(r^{\prime}=-0.44 ; r^{\prime}=-0.41 ; r^{\prime}=-0.37\right.$, respectively $)$ and positively correlated with boredom and anxiousness ( $r^{\prime}=0.37 ; r^{\prime}=0.28$, respectively). Mission duration had an adverse impact on emotions in these environmental conditions, particularly after $7 \mathrm{~h}$.

DISCUSSION: These results contribute to the understanding of fatigue development in general and of emotion-cognition relationships. These findings emphasize that both emotional states and the type of cognitive tasks to be performed should be considered for planning long-duration missions in single-piloted fighter aircrafts as to increase the probability of missions' success.

KEYWORDS: military missions, fighter aircraft, objective and subjective pilot performance.

Rosa E, Gronkvist M, Kolegard R, Dahlstrom N, Knez I, Ljung R, Willander J. Fatigue, emotion, and cognitive performance in simulated long-duration, single-piloted flight missions. Aerosp Med Hum Perform. 2021; 92(9):710-719.

L ong-duration flight missions in single-piloted aircraft are currently feasible due to increased operational capabilities of the fifth-generation aircrafts and changes in types of military operations. Air policing missions assisted by air-refueling or multiple stops can last more than $5 \mathrm{~h}$, with possible extension for more than $9 \mathrm{~h}$. Concurrently, prolonged engagement in operational tasks can lead to fatigue. ${ }^{4}$ Fatigue, in general, may impair a variety of cognitive skills and functions - such as degradation of accuracy and timing, multitasking (attention sharing), reasoning and situation awareness - and deteriorate attitude, mood and social interactions - critical for information exchange - leading to inconsistent performance. ${ }^{6}$

A challenge which arises in this domain relates to the relationship between cognition and emotions under conditions of high-stress and operational demands of long-duration $(>7 \mathrm{~h})$ flights. Emotions can affect cognition and vice-versa, exerting both transient and long-term influences, although the extent of these interactions is not yet clear ${ }^{10}$ and depends on context and types of cognitive resources in question. This is typically a complex problem, and the understanding of the cognition-emotion relationship may strengthen understanding of information processing beyond a pure cognitive task analysis. This approach contributes to recognizing performance

\footnotetext{
From the University of Gävle, Gävle, Sweden.

This manuscript was received for review in October 2020. It was accepted for publication in May 2021.

Address correspondence to: Eduardo Rosa, Ph.D., Kungsbäcksvägen 47, Hus 55, Room

310, University of Gävle, Gävle 80176. Sweden; eduardo.rosa@hig.se.

Copyright $\odot$ by The Authors.

This article is published Open Access under the CC-BY-NC license.

DOI: https://doi.org/10.3357/AMHP.5798.2021
} 
determinants in such conditions, supporting the establishment of parameters for successful planning and execution of long-duration missions.

Emotions can be conceptualized as a person's valenced (positive or negative) reactions to events, perceived as relevant to their ongoing concerns. ${ }^{21}$ Emotions are a multidimensional construct that encompasses aspects of the experiential, behavioral, and autonomic systems. ${ }^{30}$ Although there is an overlap in the literature regarding emotion and related constructs, such as mood, here we adopt a comprehensive approach, and understand emotions such as nervousness or anxiousness along with global mood states, stress, and all kinds of affective responses. ${ }^{21}$

Reciprocal interactions between emotion and cognition occur in complex dynamic behavior. ${ }^{10}$ In generic contexts, studies have indicated that moods and emotions can severely influence cognitive processes. ${ }^{9}$ For example, people may be influenced by their affective response to a target and use it as a basis for judgment. This means that people are likely to make positive evaluations and build their actions if they are happy rather than sad, facilitating performance. ${ }^{38}$

The emotion-cognition interaction has its basis in dynamic coordination of several brain regions, which often influence emotion and cognitive activity. ${ }^{1}$ This means that emotion and cognition are integrated in the brain and are sequential dynamic processes resulting from interactions of different brain circuits and their coordination in time. ${ }^{34}$ This interaction is relevant to action control regarding memory, decision-making, attention, and emotion regulation..$^{40}$ In this sense, cognitive control may be attributed to prefrontal activity inhibiting relevant subcortical emotion processing regions. ${ }^{1}$ For example, mood-congruent evaluations and the use of emotions as the basis of judgment may influence a decision process. Emotions may influence the evaluation of valenced features of the decision situation. ${ }^{37}$ In aviation, plan continuation errors - an erroneous behavior defined as a "failure to revise a flight plan despite emerging evidence suggesting it is no longer safe to proceed" ${ }^{\prime 27}$ - can be provoked by temporary impairment of a decision-making process due to associated negative emotional influences attached with a go-around flight procedure. ${ }^{7}$ This finding supports the emotion-cognition interaction in aviation and corroborates to the idea that "emotions and cognition are active processes that result in specific functional changing of the brain organization in time and the dynamical brain's response to environmental information." In the context of long-duration flight missions in the constricted cockpit environment of a single-seat aircraft, affect-mediated effects of this physical environment may play a role on the performance of cognitive processes involved, such as working memory, object representation, among other cognitive tasks.

Regarding the emotion-cognition interaction and fatigue in the aviation context, previous studies indicated significant performance decrements on basic cognitive tasks in fatigued subjects, as well as mood disturbances. ${ }^{5}$ In civil aviation, pilots who had high levels of fatigue reported significantly more cognitive and emotional disturbance than pilots with medium or low fatigue levels on long-haul international flights. ${ }^{29}$ Similarly, pilots with rest periods of less than $12 \mathrm{~h}$ in a 48 -h period had more unease and frustration. ${ }^{12}$ In military aviation, several studies have focused on circadian disruptions and continuous wakefulness on cognitive performance and self-ratings of fatigue. $^{32,39}$ Only a few studies of long-duration missions addressed fatigue during daytime operations. Neville et al. ${ }^{25}$ generally suggested that extended periods of flight duty have vaster implications for fatigue-induced errors and performance degradation than cumulative flight time of C-141 aircrews. Objective and subjective assessments of roles of fatigue and cognitive performance in 11-h flight missions indicate that performance in tasks addressing sustained attention, i.e., response time, degrades from approximately $7 \mathrm{~h}$ into the mission. ${ }^{31}$ Selfratings of fatigue correlated with response time performance.

In general terms, the nervous system is responsible for controlling the dynamics of the brain regarding emotions and cognition - or the generation and distribution of energy and resources concerning emotions, thoughts and actions. ${ }^{1}$ By adding environmental pressures into the equation, it is possible to find a parallel with the Job Demands-Resources (JD-R) model, ${ }^{2}$ appropriate in the context of this study. In the JD-R model, occupational risk factors are associated with job stress, and these can be classified in two general categories, i.e., job demands and job resources. Job demands refer to, e.g., physical and psychological aspects of the job that require sustained physical and/or psychological (cognitive and emotional) effort. Examples are high work pressure or an unfavorable physical environment. In chronic job demands involving, e.g., work overload or emotional demands, exhaustion of mental and physical resources may occur, leading to depletion of energy, i.e., state of exhaustion. ${ }^{2}$ Under demanding environmental conditions, individuals use performance-protection strategies ${ }^{16}$ which can be achieved through mobilization of sympathetic activation and/or increased subjective effort, i.e., use of active control in information processing. Thus, the greater the effort, the greater the physiological costs. Job resources refer to physical and psychological aspects of the job that may be functional for achieving work goals and/or may reduce job demands and the associated physiological and psychological costs. ${ }^{2}$

In the present context, job demands are characterized by the physical and psychological nature of the work environment and the need to mobilize resources to reach and sustain performance throughout the flight mission. Over time, participants may become fatigued as the operational context also demands continuous effort for performing cognitive tasks. Job resources are intrinsically connected to the proposed objective of the mission, that is, participants' willingness for completing the mission successfully (achieving work goals). In the simulated environment, job resources also refer to the safety of the environment (no penalty involved if not performing well), favoring distribution of energy and resources concerning emotions and cognitive processes. In the latter, individuals may use this resource to minimize the negative impact of job demands - a possible confounding factor as compared to real flight missions, which must be taken into account

The strong link between cognition and emotions, however, does not always find direct correspondence in time. This is 
because emotions may be semistatic or recurrent in time, whereas a cognitive process, by the nature of the task, must be temporary in time. ${ }^{1}$ In this sense, their relation is nonlinear. Considering this and the abovementioned aspects regarding the distribution of energy resources, the investigation of emotions throughout time may offer a broader view of individual processes, and complement the understanding of the overall demand/resources' characteristics of the mission associated with cognitive performance.

The aim of this study was to investigate the effects of long-duration missions on emotions, and their corresponding associations with cognitive performance. Regarding the two-dimensional structure of emotions, the present study explored the hypothesis that $(\mathrm{H} 1)$ ratings of positive emotions, i.e., enthusiasm, cheerfulness, and calmness, decrease as a mission progresses ${ }^{22}$ and that ratings of negative emotions, i.e., anxiousness, gloominess, and boredom, increase as a mission progresses. ${ }^{22}$ Considering selfreported fatigue ratings and emotions, the hypothesis was that, $(\mathrm{H} 2)$ as experienced fatigue increases, positive emotions decrease $^{14}$ and negative emotions increase. ${ }^{14}$ Finally, regarding associations with cognitive performance, the hypothesis was that, (H3) as cognitive performance decreases, positive emotions decrease $e^{3,23}$ and negative emotions increase. ${ }^{3,23}$ High/low activation levels were explored.

\section{METHODS}

This study is part of a larger research project on psychological effects during long-duration flight missions. Hence, the methods section is in agreement with a previous publication ${ }^{31}$ within this research project.

\section{Subjects}

Our sample consisted of 12 healthy participants ( 10 men, 2 women): 6 pilots ( 3 military flight cadets, 3 civil pilots) and 6 nonpilots. The mean (SD) age, height and body mass were 28.2 $( \pm 6.0)$ years, $179( \pm 6.8) \mathrm{cm}$, and $79.5( \pm 16.9) \mathrm{kg}$, respectively. Each subject passed a medical examination prior to being selected for the experiment. Participants were instructed that they could withdraw at any time during the experiment. Informed consent was obtained. The test procedure was in accordance with the Declaration of Helsinki and was approved by the Medical Research Ethics Review Board, in Stockholm (approval no:2018/806-3).

Experiments were performed in the Dynamic Flight Simulator - DFS (Wyle Laboratories Inc., El Segundo, CA) at the Flight Physiological Centre in Linköping, Sweden. The simulator is a centrifuge with a 9.1-m radius equipped with a high-fidelity flight simulator based on the 39 Gripen (Saab AB, Linköping, Sweden) aircraft models and controls. The flight simulator contains real 39 Gripen hardware, including a Martin-Baker seat (Martin Baker Aircraft Co. Ltd., Middlesex, UK) with a seatback angle of $28^{\circ}$, stick and throttle (Page Aerospace Ltd., Middlesex, UK), and G-valve/breathing regulator (PSU BRAG valve, Honeywell Aerospace, Yeovil, UK).
Participants were fully strapped in with a 6-point seat belt and wore a complete flight suit, which was comprised of full-coverage anti-G suit, helmet with a mask covering the mouth and nose $(116 \mathrm{H}$; Saab Ltd.), life jacket fitted with a chest bladder for assisted positive pressure breathing under high-G conditions, and gloves. Participants could urinate through the in-flight aircrew urine bladder relief system (AMXDmax ${ }^{\bowtie}$ Omni Measurement Systems, Inc, Milton, MA, USA), male and female versions, accordingly. Participants could take up to three energy bars and three protein bars and drink $1.5 \mathrm{~L}$ of water during the whole duration of the experiment. Nutritional levels were recommended by the Swedish Air Force, with a suggestion of consuming all the bars. The water amount provided was established according to the maximum volume that would fit in the Gripen fighter cockpit. Food and water intake were ad libitum. Caffeine and nicotine intake were not permitted. The DFS access door was kept closed, except during short interventions when preparing for the battery of cognitive tests. Other forms of subject/experimenter interactions occurred through radio communications. We used front and back cameras to follow participants' actions.

The experiment lasted $12 \mathrm{~h}$, counted from the beginning of the first cognitive test battery until the end of the last cognitive test battery. The time inside the DFS totaled $11 \mathrm{~h}$ (the last testing at hour 12 took place outside the DFS).

\section{Materials}

Every cognitive test battery included five cognitive tasks. Fighter pilots' operations were comprised of tasks involving low-order (nonexecutive) functions, such as sustained attention, as well as high-order (executive) functions, such as contextual cueing, short-term memory, pattern recognition, working memory and analog space representations. Cognitive tasks addressing these functions were used.

Psychomotor Vigilance Task (PVT). This task is a low-order task which targets fatigue-related changes in arousal and sustained attention deterioration associated with extended wakefulness and time on task. ${ }^{8}$ It is a simple reaction time (RT) task to stimuli, presented at random intervals. A blank screen is initially presented. Next, a fixation cross appears for $200 \mathrm{~ms}$ in the center of the screen. The fixation cross then disappears and, at random intervals, a red circle appears in the center of the screen. The participant's task was to respond to the red circle as fast as possible by pressing the space bar on a keyboard. The interstimulus interval varied between 2 and 12 s. Response latency was recorded. The duration of the task was set for $5 \mathrm{~min}$.

The following tasks are high-order tasks and target executive components of cognition.

Visual Search Task. This task evaluates saliency and contextual cueing by assessment of maintenance and tracking spatial position of objects. ${ }^{13}$ A scattered matrix of a set of letters (' $U$,' 'D,' ' $G$ ', ' $C$ ', 'Q' - distractors) and one of the letters ('X' or 'O' - target) was presented (randomly 10, 30, or 50 programmed screen load weight). Participant's task was to search for one of the two targets ('X' or 'O') - randomly presented between trials - in the 
field of distractors. Reaction time and accuracy to target detection were recorded. The task was comprised of 36 trials and lasted approximately $3.5 \mathrm{~min}$.

Match-to-Sample Task. This task assesses short-term memory and pattern recognition. ${ }^{36}$ A $6 \times 6$ matrix of red and yellow checkerboard pattern is initially presented for $1000 \mathrm{~ms}$, then it disappears for $3500 \mathrm{~ms}$. Next, two matrices appear simultaneously next to each other; the original and a second (similar) matrix. Participant's task was to select the matrix that matched the original sample. We recorded response latency and accuracy of target detection. The task was comprised of 30 trials, which lasted approximately $3 \mathrm{~min}$.

$N$-back Task. This task targets working memory (WM) function. ${ }^{18}$ It comprises random stimulus presented one by one in a sequence. Participant's task was to acknowledge whether the stimulus in the sequence matched the one that appeared in items before. We used only numbers as a stimulus type as this is characteristic of the flight environment. Three blocks were performed for each session, varying in memory load: 1-back, 2-back, and 3-back, in this order. Each stimulus (number) appeared for $500 \mathrm{~ms}$. The interstimulus interval was set to 2000 ms. Accuracy for each task was recorded. There were 50 trials per block, which was equivalent to approximately $1.5 \mathrm{~min}$ per n-back task (all three tasks lasted, then, 5 min $30 \mathrm{~s}$ ).

Mental Rotation Task. This task assesses internal representation of an object in space, which depends on imagining analog space representations. ${ }^{40}$ Initially, two figures simultaneously appeared on the screen. Participant's task was to identify whether the images were rotated or whether they were mirrored in relation to each other. Pressing the left shift button means acknowledgment that they are rotated; right shift button means acknowledgment that they are mirrored. Decision time was set to $5 \mathrm{~s}$. We recorded response latency/and accuracy for choice. The duration of the task was $3 \mathrm{~min}$.

There is a convergent view for a two-dimensional structure of pleasure/displeasure valences and high/low activation levels in investigations of current affect states in relation to cognition. ${ }^{20}$ Hence, emotions depicting pleasure-displeasure valences and high-low activation levels may be representative for associations with the cognitive tasks used, which led to the utilization of the Circumplex Affect Space instrument ${ }^{20}$ (Fig. 1) in our study. This measurement consists of eight affective states, arranged in vertical and horizontal axes of pleasure-displeasure valences and high-low levels of perceived activity. The vertical axis represents the High Activation (HA) and Low Activation (LA) levels. The horizontal axis represents the Pleasant (P) and Unpleasant (UP) valences. These define four quadrants, comprising eight states.

Each of these four quadrants is further divided in two parts each, defining another four affects, outlining the circumplex structure space. The pleasant valences are placed on the right side: Activated Pleasant (AP), Pleasant (P) and UnActivated Pleasant (UAP). The unpleasant valences are placed on the left side and they are: UnActivated Unpleasant (UAUP), UnActivated Pleasant (UP) and Activated Unpleasant (AUP).

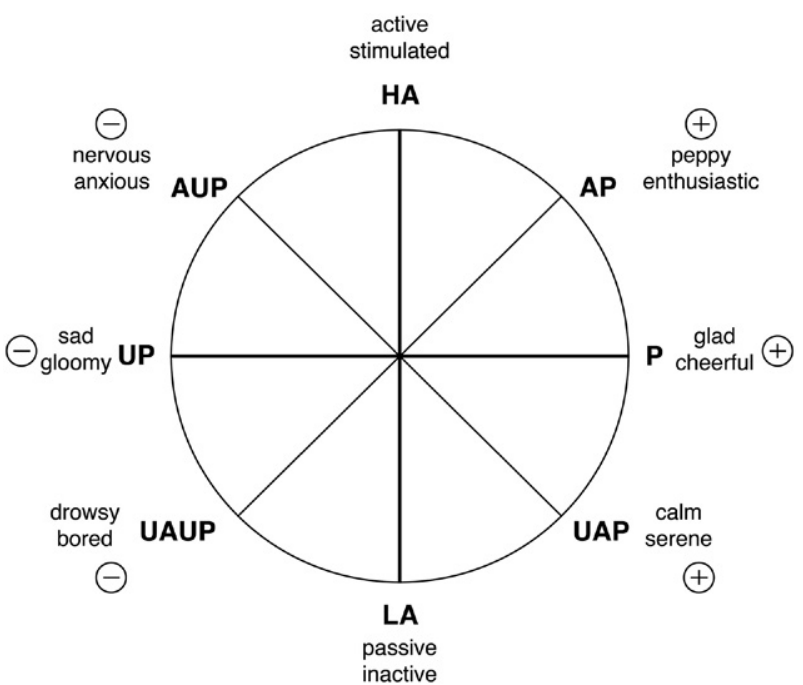

Fig. 1. The circumplex affect space, with eight affective states with two adjectives each, representing the two-dimensional structure of pleasure-displeasure and high-low levels of perceived activity. Plus and minus signs indicate positive or negative affect, respectively. Adapted from Knez. ${ }^{19}$

High activation (HA) levels are placed above, and low activation (LA) levels are placed below. They vary in magnitude of perceived activation, implying no valence.

The affect octants placed near each other $\left(45^{\circ}\right)$, e.g., HA-AP, highly associate. If they are $90^{\circ}$ from each other, e.g., AP-UAP, they have a 'near zero' association and, finally, if they are $180^{\circ}$ from each other, e.g., HA-LA, they have an 'inverse' association.

The circumplex model offers the possibility for a self-rated emotional experience at any given moment and, regarding emotion-cognition research, estimates which octant may be associated with certain cognitive task(s) performed. ${ }^{19,20}$

The two adjectives of each of the eight valences were displayed in a sheet in front of the participants, representing the short version of this task. ${ }^{19}$ Ratings were made on a 5-point scale (from 'little to not at all' to 'very much') in reply to the question 'How do you feel right now?'.

Self-reported data of fatigue were collected by the 7-point Samn-Perelli Fatigue Index. ${ }^{33}$

\section{Procedure}

Participants arrived at 7:00 a.m. at the Flight Physiological Centre. The total duration of the experiment was $12 \mathrm{~h}$. Of these 12 $\mathrm{h}$, participants remained seated inside the DFS for $11 \mathrm{~h}$. The experiment involved cognitive performance tasks and fatigue and emotion self-reported ratings (Table I).

Participants performed the cognitive test battery in six timepoints: prior to entering the DFS (hour 0), while inside the DFS (hours 3, 5, 7, and 9 - centrifuge stationary), and after they left the DFS (hour 12). We did not randomize the tasks as to deliver the same cognitive load for each task within the battery test in each time-point throughout the experiment for every participant.

The five cognitive tasks were programmed in $\mathrm{C}++$ in the open-source software system Psychology Experiment Building Language. ${ }^{24}$ A laptop computer running iOS High Sierra, 10.13 was used. The test battery was administered on a $15^{\prime \prime}$ 
Table I. The Experimental Protocol.

\begin{tabular}{|c|c|c|c|}
\hline TIME & PROCEDURE & TIME & PROCEDURE \\
\hline$\overline{0700}$ & Arrive & 1440 & Air refueling task \\
\hline 0715 & Start/Briefing & 1450 & Free flying \\
\hline 0730 & Undress & 1500 & Cognitive tests \\
\hline 0735 & Height/Weight & 1530 & Self-reported measures \\
\hline 0740 & Urine sampling & 1530 & Free flying \\
\hline 0750 & Blood sampling & 1540 & Air refueling task \\
\hline 0800 & Instrumentation (ECG) & 1550 & Free flying \\
\hline 0810 & Dressing & 1700 & Cognitive tests \\
\hline 0830 & Cognitive tests & 1730 & Self-reported measures \\
\hline 0845 & Self-reported measures & 1730 & Free flying \\
\hline 0850 & Sits in the DFS & 1740 & Air refueling task \\
\hline 0900 & Flight simulation phase starts & 1750 & Free flying \\
\hline 0915 & G-tolerance test without anti-G suit (spinning centrifuge) & 1800 & Spatial Disorientation task (spinning centrifuge) \\
\hline 0945 & G-tolerance test with anti-G suit (spinning centrifuge) & 1900 & G-tolerance test without anti-G suit (spinning centrifuge) \\
\hline 1025 & Spatial Disorientation task (spinning centrifuge) & 1930 & G-tolerance test with anti-G suit (spinning centrifuge) \\
\hline 1100 & Cognitive tests (start stationary centrifuge time) & 2000 & Flight simulation phase ends \\
\hline 1130 & Self-reported measures & 2000 & Moving out from the DFS \\
\hline 1135 & Free flying & 2005 & Undress \\
\hline 1240 & Air refueling task & 2010 & Urine sampling \\
\hline 1250 & Free flying & 2020 & Blood sampling \\
\hline 1300 & Cognitive tests & 2030 & Cognitive tests \\
\hline 1330 & Self-reported measures & 2055 & Self-reported measures/Debrief \\
\hline 1335 & Free flying & 2100 & Weight/Release \\
\hline
\end{tabular}

MacBook Pro for the hour 0 and hour 12 (outside the DFS) and were mirrored to the central DFS' $20^{\prime \prime}$ LCD monitor for the hours 3, 5, 7 and 9 (inside the DFS). Participants used a wired keyboard with an integrated trackball on top of an adapted lap support in the DFS.

After each cognitive test battery, participants rated emotions by the self-reported affect circumplex instrument and perceived fatigue by the SPFI. Ratings were made by reading the corresponding scales, which had been taped inside the DFS gondola and conveyed through the intercommunication system.

Participants were allowed to engage in 'free flying' in the simulator between the cognitive tests, as depicted in Table I.

\section{Statistical Analyses}

We used a within-subjects design for studying changes of fatigue, emotions, and cognitive performance across time. The dependent variables were the performance in cognitive tests and self-reported ratings of fatigue and emotions. Post hoc differences were determined using Bonferroni multiple-comparison correction. A $P<0.05$ level of significance was used to determine statistical differences for all analyses. Furthermore, we used correlational analyses to calculate the positive/negative associations of fatigue, emotions, and cognitive performance. A correlational design was used to measure the strength and direction of these associations and, due to the low number of participants, prevent a between-subject analysis.

\section{RESULTS}

Overall fatigue ratings and cognitive performance results are described below. We then report the results related to our three hypotheses.
Regarding self-reported fatigue (and as reported elsewhere ${ }^{31}$ ), the Samn-Perelli Fatigue Index showed that perceived fatigue increased over time, $F(5,50)=11.66, P<0.001, \eta_{\mathrm{p}}{ }^{2}=$ 0.53 . Bonferroni post hoc tests revealed a difference between hour 0 and hour $7(P=0.04$, Mean difference $95 \%$ CI $[-3.2$, $-0.59])$, between hour 0 and hour $9(P=0.02$, Mean difference $95 \% \mathrm{CI}[-3.78,-0.21])$ and between hour 0 and hour $12(P=$ 0.002, Mean difference 95\% CI [-3.74, -0.80]) (Fig. 2A).

Regarding cognitive performance, the PVT task revealed an increase in response time over the duration of the experiment, $F(5,50)=7.87, P=0.001, \eta_{\mathrm{p}}{ }^{2}=0.44$ (Fig. 2B). Bonferroni post hoc tests revealed that a difference occurred between hour 0 and hour $7[P=0.02$, Mean difference $95 \%$ CI $(-75.6,-5.3)]$, between hour 0 and hour $9[P=0.01$, Mean difference $95 \%$ CI $(-76.9,-7.8)]$ and between hour 0 and hour $12[P=0.01$, Mean difference $95 \%$ CI $(-38.04,-3.4)]$.

No other significant results on accuracy or reaction time were found for the high-order cognitive tasks throughout the $12 \mathrm{~h}$ of the experiment.

Regarding High Activation (HA) and Low Activation (LA) levels over time, high activation level results indicated that participants were significantly less active/stimulated, $F(5,50)=$ 11.7, $P<0.001, \eta_{\mathrm{p}}{ }^{2}=0.54$, over time. Bonferroni post hoc tests for active/stimulated revealed a difference between hour 0 and hour $7[P=0.005$, Mean difference $95 \% \mathrm{CI}(0.45,2.82)]$, hour 0 and hour $9(P=0.001$, Mean difference $95 \%$ CI $[0.24,2.84])$ and between hour 0 and hour $12[P=0.002$, Mean difference 95\% CI $(0.57,2.70)]$. Low activation (LA) level results showed that participants were significantly more passive/inactive with time, $F(5,50)=4.17, P<0.05, \eta_{\mathrm{p}}{ }^{2}=0.29$. Bonferroni pairwise multiple comparisons tests, however, did not reveal significant differences (Fig. 3). 

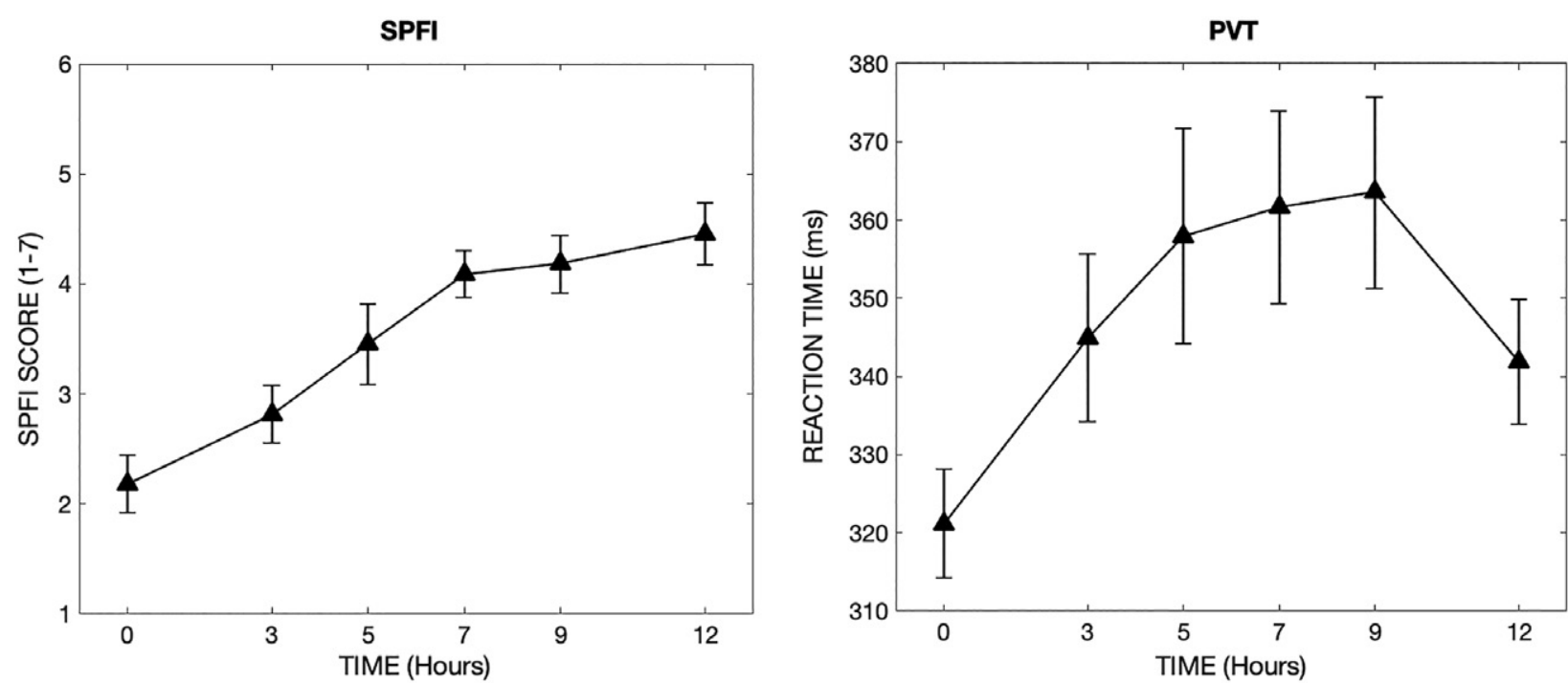

Fig. 2. Means ( \pm SE) for: A. (left) self-reported measure of fatigue determined by the Samn-Perelli Fatigue Index; and B. (right) the PVT task over time.

Hypothesis 1 addressed ratings of positive emotions and ratings of negative emotions over time. Circumplex Affect Space results indicated that Activated Pleasant (AP) and Pleasant (P) valences were affected over time; participants were significantly less peppy/enthusiastic, $F(5,50)=14.1, P<0.001, \eta_{\mathrm{p}}^{2}=0.58$, and less glad/cheerful, $F(5,50)=8.4, P<0.001, \eta_{\mathrm{p}}{ }^{2}=0.45$, respectively (Fig. 4).

Bonferroni post hoc tests for peppy/enthusiastic revealed a difference between hour 0 and hour $7[P=0.009$, Mean difference $95 \%$ CI $(0.35,2.74)]$, between hour 0 and hour 9 $[P=0.002$, Mean difference $95 \% \mathrm{CI}(0.57,2.70)]$ and between hour 0 and hour $12[P=0.00$, Mean difference $95 \% \mathrm{CI}$ $(0.56,2.89)]$.

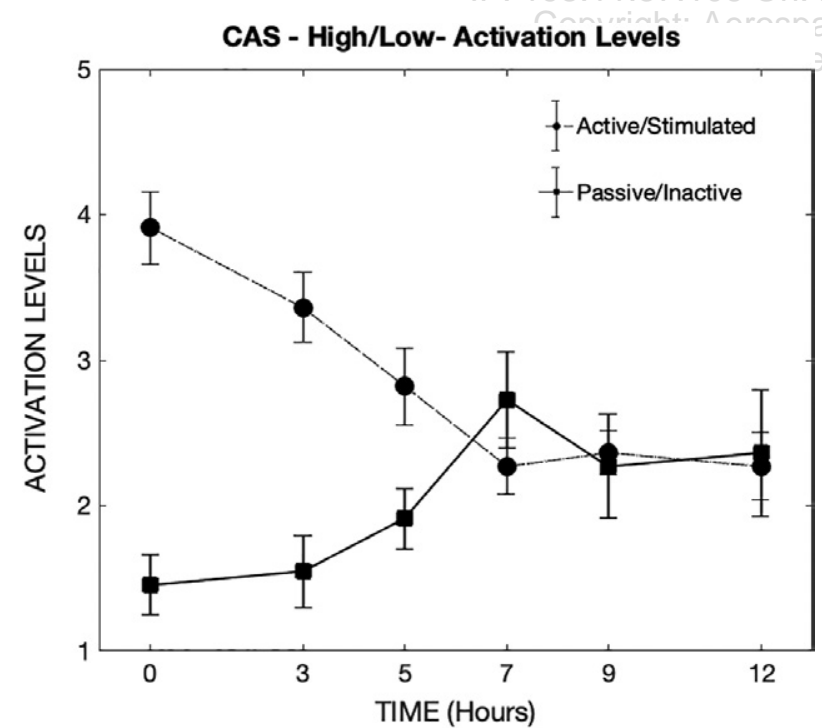

Fig. 3. Circumplex Affect Space results depicting the Mean ( \pm SE) activation levels throughout the 12-h. Participants were significantly less active/stimulated and significantly more passive/inactive overall (global effect), although for this emotional state we did not find significant differences after performing multiple pairwise comparisons.
Bonferroni post hoc tests for glad/cheerful revealed a difference between hour 0 and hour $7[P=0.02$, Mean difference $95 \%$ $\mathrm{CI}(0.10,2.43)]$ and between hour 0 and hour $9[P=0.05$, Mean difference $95 \%$ CI $(0.002,2.18)]$.

The emotional state addressing the UnActivated Pleasant (UAP) valence (i.e., calm/serene, ) did not show any significant difference over time, $F(5,50)=1.33, P=0.26, \eta_{\mathrm{p}}{ }^{2}=0.11$.

Regarding negative emotions, the UnActivated Unpleasant (UAUP) valence also revealed significant differences over time; participants were significantly more drowsy/bored, $F(5,50)=$ 8.5, $P<0.001, \eta_{\mathrm{p}}{ }^{2}=0.46$ (Fig. 5). Bonferroni post hoc tests revealed a difference between hour 0 and hour $7(P=0.001$, Mean difference $95 \%$ CI $[-2.77,-0.68])$, between hour 0 and
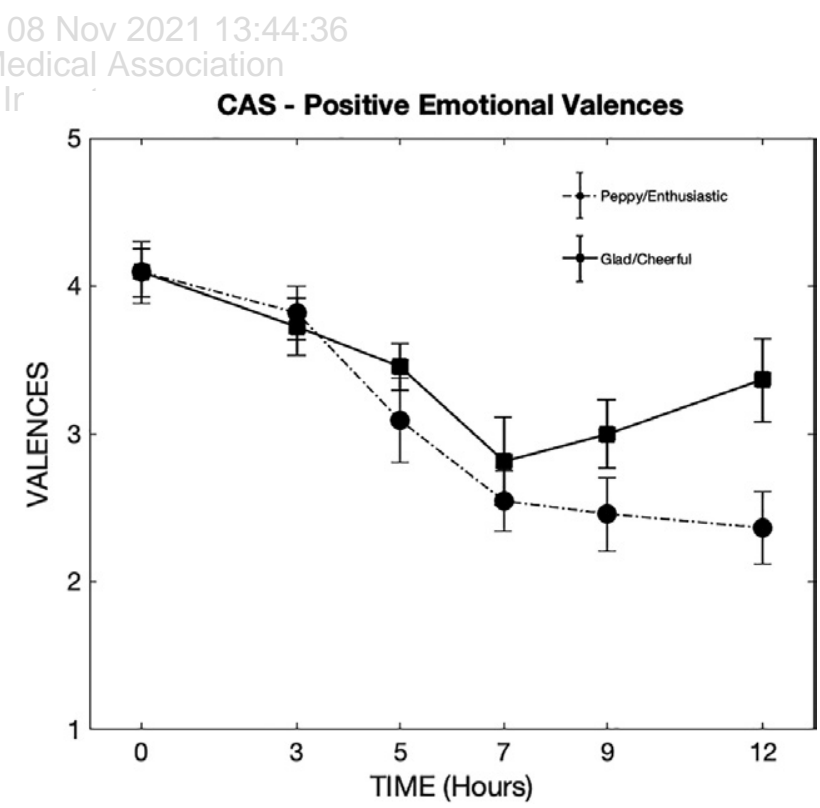

Fig. 4. Circumplex Affect Space results depicting the Mean ( \pm SE) of the two significant positive emotional-state differences over the 12-h. Participants were significantly less peppy/enthusiastic and significantly less glad/cheerful from approximately $7 \mathrm{~h}$ into the mission. 


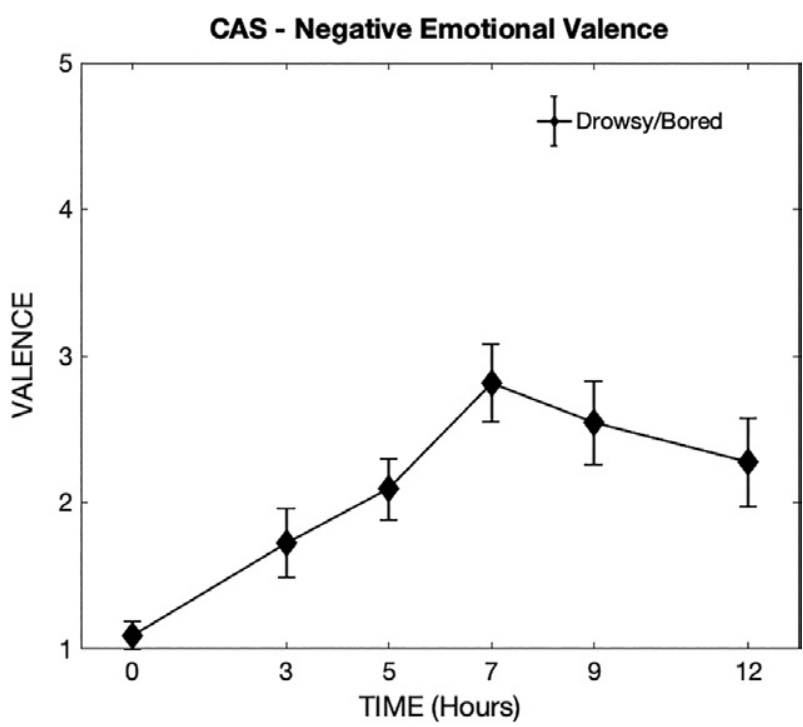

Fig. 5. Circumplex Affect Space results depicting the Mean ( \pm SE) of the significant negative emotional-state differences over the 12-h. Participants were significantly drowsier/bored from approximately $7 \mathrm{~h}$ into the mission.

hour $9(P=0.006$, Mean difference $95 \% \mathrm{CI}[-2.53,-0.37])$ and between hour 0 and hour $12(P=0.003$, Mean difference $95 \%$ CI $[-2.31,-0.04])$.

Emotional states addressing Unpleasant (UP) and Activated Unpleasant (AUP) valences, i.e., sad/gloomy, nervous/anxious, respectively, did not show any significant difference over time, $F(5,50)=0.95, P=0.45, \eta_{\mathrm{p}}{ }^{2}=0.08$ and $F(5,50)=1.41, P=0.23$, $\eta_{\mathrm{p}}^{2}=0.12$, respectively.

Taken together, these results reveal a decrease in the positive emotional ratings addressing enthusiasm and cheerfulness and an increase in the negative emotional rating addressing boredom after approximately $7 \mathrm{~h}$ into the mission. These ratings did not improve until the end of the mission.

Hypothesis 2 addressed experienced fatigue and associations with positive and negative emotions. There was a significant negative correlation between experienced fatigue (SPFI) and the positive affect states in the Activated Pleasant (AP) and Pleasant $(\mathrm{P})$ valences, i.e., peppy/enthusiastic, glad/cheerful, $\left(r^{\prime}=-0.75, N=11, P<0.001 ; r^{\prime}=-0.49, N=11, P<0.001\right.$, respectively).

Regarding negative emotions, there was a significant positive correlation between experienced fatigue (SPFI) and the UnActivated Unpleasant and Unpleasant valences, i.e., drowsy/ bored and sad/gloomy, respectively, $\left(r^{\prime}=0.61, N=11, P<0.001\right.$; $\left.r^{\prime}=0.30, N=11, P<0.05\right)$.

Hence, as experienced fatigue increases, positive emotions addressing enthusiasm and cheerfulness decrease and negative emotions addressing boredom and gloominess increase.

Finally, hypothesis 3 considered cognitive performance and associations with positive and negative emotions. There was a negative correlation between the Activated Pleasant (AP), Pleasant $(\mathrm{P})$ and UnActivated Pleasant valences (i.e., peppy/enthusiastic, glad/cheerful and calm/serene) and increased response times in PVT - correlation coefficients $\left(r^{\prime}=-0.44, N=11, P<0.001 ; r^{\prime}=-0.41, N=11, P<0.001\right.$;
Table II. Correlations Between Performance in the PVT Task, SPFI and the Eight Emotional States.

\begin{tabular}{lrrcc}
\hline MEASURES & M & SD & PVT_RT & SPFI \\
\hline PVT_RT & 348.53 & 37.88 & - & \\
SPFI & 3.53 & 1.21 & $0.291^{*}$ & - \\
Peppy/enthusiastic & 3.06 & 1.01 & $-0.441^{* *}$ & $-0.750^{* *}$ \\
Glad/cheerful & 3.41 & 0.84 & $-0.412^{* *}$ & $-0.491^{* *}$ \\
Calm/serene & 3.73 & 1.03 & $-0.370^{* *}$ & -0.167 \\
Nervous/anxious & 1.23 & 0.49 & $0.286^{*}$ & 0.027 \\
Sad/gloomy & 1.20 & 0.59 & 0.148 & $0.306^{*}$ \\
Drowsy/bored & 2.09 & 0.96 & $0.377^{* *}$ & $0.611^{* *}$ \\
Active/stimulated & 2.83 & 0.95 & $-0.446^{* *}$ & $-0.684^{* *}$ \\
Passive/inactive & 2.05 & 1.09 & $0.301^{*}$ & $0.380^{* *}$ \\
\hline
\end{tabular}

$r^{\prime}=-0.37, N=11, P<0.001$, respectively). It is important to note that these correlations indicate a positive association between cognitive performance and positive affect states, that is, as cognitive performance decreases in this task, so as positive emotions

Regarding negative emotions, there was a significant positive correlation between the UnActivated/Unpleasant (UAUP), and Activated Unpleasant (AUP) valences (i.e., drowsy/bored and nervous/anxious, respectively) and increased response times in PVT - correlation coefficients $\left(r^{\prime}=0.37, N=11\right.$, $P<0.001 ; r^{\prime}=0.28, N=11, P<0.05$, respectively). Similarly, it is important to note that these correlations indicate a negative association between cognitive performance and negative emotions, that is, as cognitive performance decreases in this task, negative emotions increase.

In summary, as cognitive performance in the task addressing sustained attention decreases, positive emotions indicating enthusiasm, cheerfulness and calmness decrease, and negative emotions indicating boredom and anxiety increase.

Associations between experienced fatigue, cognitive performance and High Activation (HA), Low Activation (LA) levels were explored. There was a significant negative correlation between experienced fatigue and the High Activation (HA) levels (active/stimulated) $\left(r^{\prime}=-0.68, N=11, P<0.001\right)$. The correlation was also observed with increased response times in $\operatorname{PVT}\left(r^{\prime}=-0.44, N=11, P<0.001\right)$.

There was a significant positive correlation between experienced fatigue and the Low Activation (LA) levels (passive/inactive) $\left(r^{\prime}=0.38, N=11, P<0.002\right)$. There was also a significant positive correlation with increased response times in PVT $\left(r^{\prime}=0.30, N=11, P<0.05\right)$.

Combined results indicate that as experienced fatigue increases, participants are less active and more inactive. Similarly, as sustained attention decreases, participants were less active and more inactive.

Table II depicts the correlations between the positive and negative valences, the High Activation and Low Activation levels and the abovementioned variables related to $\mathrm{H} 2$ and $\mathrm{H} 3$.

After Bonferroni correction to control for the familywise error rate we found no significant correlations between the emotions and the high-order cognitive measures. 


\section{DISCUSSION}

The aim of this study was to investigate the effects of long-duration missions on emotions and the correlations with cognitive performance in pilots. More specifically, we analyzed variations in emotions across time and the associations between emotions and cognitive performance. Results revealed that positive emotions addressing enthusiasm and cheerfulness significantly decreased after approximately $7 \mathrm{~h}$ into the mission, and remained significantly lower, compared to the initial self-reported ratings, until the end of the experiment at hour 12. The only negative emotion that was significantly affected was the feeling of being bored, and this emotional state remained significantly higher until the end of the experiment at hour 12 . These particular self-reported emotional ratings significantly correlated with experienced fatigue. These results partially confirmed hypothesis 1 - that positive emotions would decrease and negative emotions would increase over time - and hypothesis 2 - which stated that, as experienced fatigue increases, positive emotions decrease and negative emotions increase. Regarding cognitive performance associations with emotional states, hypothesis 3 stated that, as cognitive performance decreases, positive emotions decrease and negative emotions increase. There were significant associations between the low-order task performance only, implying that, as sustained attention decreases, enthusiasm, cheerfulness, and calmness decrease, and boredom and anxiousness increase. Finally, regarding high- and low-activation levels, participants were significantly less active and more inactive also approximately $7 \mathrm{~h}$ into the mission. Moreover, correlations revealed that, as experienced fatigue increases and sustained attention decreases, participants were significantly less active and more inactive. High-order tasks were not affected or associated with experienced fatigue or emotional ratings.

The Circumplex Affect Space also indicates how the dimensions are associated between the affect octants, that is: 1) high correlation when located nearby (e.g., HA-AP); 2) near zero, when $90^{\circ}$ from each other (e.g., HA-P); and 3) inversely, when $180^{\circ}$ from each other (e.g., HA-LA). In this context, the results showed that perceived high activation (HA) change across time valenced with pleasantness (AP); both decreased as the mission progressed. Correspondingly, perceived low activation (LA) change across time valenced with the unpleasantness (UAUP); both increased as the mission progressed.

These results allow interpretations of the reciprocal influences observed in emotion-cognition interactions, as described by Dolcos et al. ${ }^{10}$ Regarding the impact of emotion on cognition, one position supports the view that emotion processing depends on available attentional resources. ${ }^{28}$ Task context may affect emotional responses to cognitive challenges, and an emotional situation may evoke a wide range of emotional responses across individuals. ${ }^{10}$ Negative emotions and increased susceptibility to emotional distraction are accompanied by overall impairment cognitive/executive control, affecting high-order cognitive control brain regions and low-order perceptual processing regions. ${ }^{10}$ In the opposing direction, research on the effect of cognition on emotion addresses the importance of cognitive control of emotion (or emotion regulation) in the presence of emotional stressors. Studies have focused on conscious forms of emotion regulation, such as reappraisal, i.e., the cognitive transformation of an emotional experience, ${ }^{26}$ and suppression, i.e., the deliberate effort to suppress undesirable emotional responses. ${ }^{17}$ As such, self-regulation involves executive control, and the capacity to self-regulate depends on the energy resources available at the time. ${ }^{35}$ On the neurobiological level, cognitive control may require prefrontal activity inhibiting related subcortical emotion processing regions. ${ }^{1}$

As emotion and cognition are understood as dynamic processes rising from interaction of several brain subsystems and depends on their coordination in time ${ }^{34}$ the nervous system plays a role in controlling the generation and distribution of the energy and resources between emotions, thoughts and actions. ${ }^{1}$ Hence, in general, the dynamic influences between emotion and cognition suggest that emotion can impair cognitive control due to depletion of attentional resources and, conversely, the depletion of energy resources for cognitive control can impair emotion regulation.

Considering the direction of the emotion-cognition association, it may be the case that emotions played a role in the cognitive results. We did not observe a decrease in performance in the high-order tasks. However, significant correlations between a decrease in the low-order task performance, the high- and low-activation levels, the three positive valences and one negative valence seem to indicate that a depletion of attentional resources played a role in this paradigm. Increase of response time is generally associated with fatigue ${ }^{39}$ and PVT results are generally interpreted as reflecting the arousal and attentional state of the individual. ${ }^{11}$ Slow response time in the PVT task may be characterized by activation within the default-mode network, suggestive of inattention and task disengagement. ${ }^{11}$ Considering this, it seems reasonable to assume that a decrease of attentional resources is at play in the observed associations, indicating a decrease in overall arousal among participants - as observed by a decrease in activeness, enthusiasm and cheerfulness, an increase in boredom and passiveness - as well as task disengagement, after approximately $7 \mathrm{~h}$ into the mission.

A similar reasoning can be pondered for the direction of the cognition-emotion association. Emotional levels and valences observed were affected after approximately $7 \mathrm{~h}$, as well as performance in the PVT, and these did not improve until the end of the mission. It is possible to assume there was a reduction of available energy resources around this point in time, impacting cognitive control over behavior. Since emotion regulation is, by definition, a control process, ${ }^{21}$ this subsequentially affected some deliberative form of emotion regulation. These results agree with the dynamics of the brain pointed by Afraimovich ${ }^{1}$ earlier, stating that the supply and allocation of energy and resources concerning emotions, thoughts and actions is governed by the nervous system, which is constantly seeking for its internal regulation. Recently, Grillon et al. ${ }^{15}$ pointed out that mental fatigue, i.e., depletion of cognitive resources - or the extent to which these resources get exhausted and recover over 
time - can impair emotion regulation. We interpret the findings of our study following this approach; prior consumption of cognitive resources impaired subsequent emotion regulation.

It is important to emphasize, however, that emotion regulation generally involves the $\mathrm{PFC},{ }^{26}$ which also supports other forms of cognitive control. In this study, as mentioned, decrease in performance of high-order tasks or association with emotions were not observed. The most plausible interpretation for difficulties in self-regulation of emotions may relate to the incongruence between the perception of available energy resources and for accomplishing the objective, that is, sustaining performance until the end of the experiment. The JD-R model notes that individuals may use performance-protection strategies to sustain performance, such as active use of information processing. Sustaining performance of high-order tasks could have been possible due to increased activation and/or subjective effort. Energy resources, however, were drained, compromising emotion regulation. This interpretation also finds support in the cognitive-energy model posed by Zohar et al. $^{41}$ which posits that the levels of energy available for selfregulation will influence the intensity of emotional reaction, as disruptive of challenging circumstances require effortful self-regulation. In the military aviation context, results from this study are also in line with Caldwell et al., ${ }^{5}$ indicating that fatigue-induced mood impairments of F-117A pilots can increase the amount of perceived effort required to accomplish mission objectives.

One of the limitations of this study concerns the recruitment of participants; restrictions prevented us having only pilots in our sample. Of the six pilots included as participants, three were Swedish Air Force pilots under training, i.e., they had not started flying the 39 Gripen fighter. Hence, the study design was centered on the information processing view of cognition, i.e., mental processes, representative of the natural environment. Although we have used a full-flight simulator environment, this is different from more ecological approaches to cognition and in using real flight tasks (full-flight simulations), where the investigation includes the practical value of any associated mental construct.

Hence, in assessing the low-order task, we are aware of the fact that cognitive energy costs for sustaining attention in a simulator is lower than in a real flight, which may indicate differences in time in performance degradation. This may also be the case for the high-order executive tasks used; the tasks might not have been complex enough for us to observe decreased performance. We suggest that the results should not be interpreted as tasks taxing high-order functions are not affected by the effects of fatigue in any circumstance. Rather, performance differences in these tasks are harder to detect than in tasks taxing low-order functions. Similarly, emotional ratings in a real situation may vary, as energy resources for emotion regulation in a more unstable environment would presumably differ from a simulator study. Investigation of sympathetic/parasympathetic activation associated with emotional ratings could clarify these potential differences.

In this sense, although cognitive performance deterioration and self-reported perception of fatigue is in accordance with previous studies, it is not an easy task to transfer the results from our tests to real flights. Nevertheless, the current study indicates that both positive and negative emotions may be influenced by mission duration. In general, emotions significantly correlate with subjective perception of fatigue and with performance of a nonexecutive cognitive task. Decrease in positive emotions and increase of negative emotions can reflect increases of fatigue, particularly after $7 \mathrm{~h}$ into the mission.

Combined results suggest that pilots may be able to adapt to environmental stressors in long-duration missions so long as available energy resources match the demands associated with mission objectives. This allows cognitive control over behavior, favoring emotion regulation and protecting performance until the end of the mission.

Integrating emotions and cognitive results may offer a broader understanding of pilot performance in challenging conditions. From the operational point of view, pilot's awareness and report of emotional states can be useful for indicating fatigue development. Hence, emotional ratings could also be used for performance monitoring and, as indicated in this study, a self-reported two-dimensional structure of current affect instrument is adequate. Emotions and cognitive functions are integral parts of a larger computational system, and observing this system in a wider perspective may result in a better understanding of the interaction between the pilot and the mission.

\section{ACKNOWLEDGMENTS}

This study was supported by the Swedish Armed Forces grant no 922:0918. The Ethics Application for the experiments is $n^{\circ}$ 2018/806-31, "Fysiologiska och kognitiva effekter av långvariga flygpass i JAS39Gripen". We are grateful to the staff and engineers of the Dynamic Flight Simulator at the Flight Physiological Center for their kind assistance in the study.

Financial Disclosure Statement: The authors listed certify that they have no affiliations or relationships with any individuals, organizations or entities with any financial or non-financial interests in the subject matter addressed in the submitted manuscript.

Authors and Affiliations: Eduardo Rosa, Ph.D., Igor Knez, Ph.D., and Johan Willander, Ph.D., Department of Psychology, and Robert Ljung, Ph.D., Department of Environmental Psychology, University of Gävle, Gävle, Sweden; Mikael Grönkvist, Ph.D., and Roger Kölegård, Ph.D., Division of Environmental Physiology, Swedish Aerospace Physiology Center, KTH Royal Institute of Technology, Stockholm, Sweden; and Nicklas Dahlström, Ph.D., Lund University School of Aviation, Lund University, Lund, Sweden.

\section{REFERENCES}

1. Afraimovich V, Young T, Muezzinoglu MK, Rabinovich MI. Nonlinear dynamics of emotion-cognition interaction: When emotion does not destroy cognition? Bull Math Biol. 2011; 73(2):266-284.

2. Bakker AB, Demerouti E. The Job Demands-Resources model: State of the art. Journal of Managerial Psychology. 2007; 22(3):309-328.

3. Bolmont B, Thullier F, Abraini JH. Relationships between mood states and performances in reaction time, psychomotor ability, and mental efficiency during a 31-day gradual decompression in a hypobaric chamber from sea level to $8848 \mathrm{~m}$ equivalent altitude. Physiol Behav. 2000; 71(5):469-476 
4. Bourgeois-Bougrine S, Carbon P, Gounelle C, Mollard R, Coblentz A. Perceived fatigue for short-and long-haul flights: a survey of 739 airline pilots. Aviat Space Environ Med. 2003; 74(10):1072-1077.

5. Caldwell JA, Caldwell JL, Brown DL, Smith JK. The effects of 37 hours of continuous wakefulness on the physiological arousal, cognitive performance, self-reported mood, and simulator flight performance of F-117A pilots. Mil Psychol. 2004; 16(3):163-181.

6. Caldwell JA, Caldwell JL, Schmidt RM. Alertness management strategies for operational contexts. Sleep Med Rev. 2008; 12(4):257-273.

7. Causse M, Dehais F, Péran P, Sabatini U, Pastor J. The effects of emotion on pilot decision-making: a neuroergonomic approach to aviation safety. Transp Res Rec. 2013; 33:272-281.

8. Dinges DF, Powell JW. Microcomputer analyses of performance on a portable, simple visual RT task during sustained operations. Behav Res Methods Instrum Comput. 1985; 17(6):652-655.

9. D'Mello SK, Lehman B, Person N. Monitoring affect states during effortful problem solving activities. Int J Artif Intell Educ. 2010; 20(4):361-389.

10. Dolcos F, Iordan AD, Dolcos S. Neural correlates of emotion-cognition interactions: A review of evidence from brain imaging investigations. J Cogn Psychol (Hove). 2011; 23(6):669-694.

11. Drummond SP, Bischoff-Grethe A, Dinges DF, Ayalon L, Mednick SC, Meloy MJ. The neural basis of the psychomotor vigilance task. Sleep. 2005; 28(9):1059-1068.

12. Drury DA, Ferguson SA, Thomas MJW. Restricted sleep and negative affective states in commercial pilots during short haul operations. Accid Anal Prev. 2012; 45:80-84.

13. Eckstein MP. Visual search: A retrospective. J Vis. 2011; 11(5):14.

14. French J, Bisson RU, Neville KJ, Mitcha J, Storm WF. Crew fatigue during simulated, long duration B-1B bomber missions. Aviat Space Environ Med. 1994; 65(5):A1-A6.

15. Grillon C, Quispe-Escudero D, Mathur A, Ernst M. Mental fatigue impairs emotion regulation. Emotion. 2015; 15(3):383-389.

16. Hockey GRJ. Cognitive-energetical control mechanisms in the management of work demands and psychological health. In: Baddely A, Weiskrantz L, editors. Attention: Selection, Awareness, and Control. Oxford, UK: Clarendon Press; 1993:328-345.

17. Jackson DC, Mueller CJ, Dolski I, Dalton KM, Nitschke JB, et al. Now you feel it, now you don't: Frontal brain electrical asymmetry and individual differences in emotion regulation. Psychol Sci. 2003; 14(6):612-617.

18. Kane MJ, Conway ARA, Miura TK, Colflesh GJH. Working memory, attention control, and the n-back task: A question of construct validity. J Exp Psychol Learn Mem Cogn. 2007; 33(3):615-622. ght: Aerospa

19. Knez I. Affective and cognitive reactions to subliminal flicker from fluorescent lighting. Conscious Cogn. 2014; 26:97-104.

20. Knez I, Hygge S. The circumplex structure of affect: A Swedish version. Scand J Psychol. 2001; 42(5):389-398.

21. Koole SL, Van Dillen LF, Sheppes G. The self-regulation of emotion. Handbook of self-regulation: Research, theory, and applications. New York (NY): Guilford Press; 2011:22-40.

22. Lieberman HR, Bathalon GP, Falco CM, Kramer FM, Morgan CA, Niro P. Severe decrements in cognition function and mood induced by sleep loss, heat, dehydration, and undernutrition during simulated combat. Biol Psychiatry. 2005; 57(4):422-429.

23. Lieberman HR, Tharion WJ, Nindl BC, Castellani JW, Montain SJ. Cognition during sustained operations: comparison of a laboratory simulation to field studies. Aviat Space Environ Med. 2006; 77(9):929-935.

24. Mueller ST, Piper BJ. The Psychology Experiment Building Language (PEBL) and PEBL Test Battery. J Neurosci Methods. 2014; 222:250-259.

25. Neville KJ, Bisson RU, French J, Boll PA, Storm WF. Subjective fatigue of C-141 aircrews during operation desert storm. Hum Factors. 1994; 36(2):339-349.

26. Ochsner KN, Bunge SA, Gross JJ, Gabrieli JD. Rethinking feelings: an FMRI study of the cognitive regulation of emotion. J Cogn Neurosci. 2002; 14(8):1215-1229.

27. Orasanu J, Martin L, Davison J. Cognitive and contextual factors in aviation: Decision errors. In: Salas E, Klein G, editors. Linking expertise and naturalistic decision making. Sussex, UK: Psychology Press; 2001:209-222.

28. Pessoa L. To what extent are emotional visual stimuli processed without attention and awareness? Curr Opin Neurobiol. 2005; 15(2):188-196.

29. Petrie KJ, Dawson AG. Symptoms of fatigue and coping strategies in international pilots. Int J Aviat Psychol. 1997; 7(3):251-258.

30. Pinna T, Edwards DJ. A systematic review of associations between interoception, vagal tone, and emotional regulation: Potential applications for mental health, wellbeing, psychological flexibility, and chronic conditions. Front Psychol. 2020; 11:1792.

31. Rosa E, Eiken O, Grönkvist M, Kölegård R, Dahlström N, et al. Effects of fatigue on cognitive performance in long-duration simulated flight missions. Aviat Psychol Appl Hum Factors. 2020; 10(2):82-93.

32. Russo MB, Kendall AP, Johnson DE, Sing HC, Thorne DR, et al. Visual perception, psychomotor performance, and complex motor performance during an overnight air refueling simulated flight. Aviat Space Environ Med. 2005; 76(7):C92-C103.

33. Samn SW, Perelli LP.Estimating aircrew fatigue: a technique with application to airlift operations. School of Aerospace Medicine Brooks AFB, TX; 1982.[Accessed 24 June 2021.] Available from: https://apps.dtic.mil/sti/ pdfs/ADA125319.pdf.

34. Scherer KR. Neuroscience projections to current debates in emotion psychology. Cogn Emotion. 1993; 7(1):1-41.

35. Schmeichel BJ. Attention control, memory updating, and emotion regulation temporarily reduce the capacity for executive control. J Exp Psychol Gen. 2007; 136(2):241-255.

36. Shurtleff D, Thomas JR, Schrot J, Kowalski K, Harford R. Tyrosine reverses a cold-induced working memory deficit in humans. Pharmacol Biochem Behav. 1994; 47(4):935-941.

37. Schwarz N. Emotion, cognition, and decision making. Cogn Emotion. 2000; 14(4):433-440.

38. Tenenbaum G, Edmonds WA, Eccles DW. Emotions, coping strategies, and performance: A conceptual framework for defining affect-related performance zones. Mil Psychol. 2008; 20(suppl.1):S11-S37.

39. Van Dongen HPA, Caldwell JA, Caldwell JL. Investigating systematic individual differences in sleep-deprived performance on a high-fidelity flight simulator. Behav Res Methods. 2006; 38(2):333-343.

40. Zacks JM. Neuroimaging studies of mental rotation: a meta-analysis and review. J Cogn Neurosci. 2008; 20(1):1-19.

41. Zohar D, Tzischinski O, Epstein R. Effects of energy availability on immediate and delayed emotional reactions to work events. J Appl Psychol. 2003; 88(6):1082-1093 